\title{
VAissié Cécile, Le Clan Mikhalkov. Culture et pouvoirs en Russie (1917-2017), Presses universitaires de Rennes, Rennes, 2019, 398 p.
}

Catherine GÉRY

Le Clan Mikhalkov. Culture et pouvoirs en Russie (1917-2017) est une contre-enquête ou un contre-récit à l'histoire des Mikhalkov « racontée par eux-mêmes »- leur story, pour reprendre Christian Salmon dans son ouvrage sur le storytelling ou la machine à fabriquer des histoires. Cette contre-enquête, Cécile Vaissié l'a rédigée suite à de longues et patientes recherches sur une des familles parmi les plus célèbres de Russie et d'URSS, et sur les relations complexes mais constantes que cette famille a entretenues et continue d'entretenir avec les pouvoirs. La thèse développée dans l'ouvrage est que cette histoire familiale ou cette story s'est construite en miroir de l'histoire politique, culturelle et sociale dans laquelle elle s'inscrit. La méthodologie suivie par l'A. reprend donc de manière réflexive et distanciée l'amalgame cultivé par Nikita Mikhalkov entre le destin des membres de sa famille et celui de la Russie : elle consiste à articuler des « parcours individuels » aux parcours de « groupes sociaux » et à l'histoire «d'un pays tout entier », une histoire qui croise d'ailleurs aussi... celle de l'Inalco (Viktoria, demi-sœur du peintre Piotr Kontchalovski, a enseigné le russe aux Langues O' de 1907 à 1954).

Une grande partie des historiens ou des sociologues s'accordent aujourd'hui sur le fait que tout parcours individuel s'insère dans une histoire collective dont il peut témoigner, et que le discours sur soi constitue un biais pertinent pour appréhender, au-delà de la construction identitaire individuelle, le sens de phénomènes tels que les trajectoires sociales ou les changements culturels et historiques. Mais s'il est devenu habituel pour l'historien contemporain, le postulat qui veut qu'en produisant des 
SLOVO

histoires, la narration de soi produit aussi de l'Histoire, prend ici une dimension inédite et passionnante. En effet, les membres de la famille Mikhalkov n'ont eu de cesse d'élaborer à partir de leur propre vie ce que l'A. appelle un « conte russe », c'est-à-dire une fiction au sens russe du terme: quelque chose de contraire à la réalité, un pur produit de l'imagination, une illusion, un dispositif de leurre. Sur le site de Mediapart, Jean-Pierre Thibaudat parle d'ailleurs joliment du « soleil trompeur des Mikhalkov » dans sa recension du livre. Aussi, la question posée par Le clan Mikhalkov est la suivante : que peut nous apprendre la fiction d'une famille sur les réalités de la Russie et les mécanismes du pouvoir?

J'aimerais ici souligner l'importance d'une toute petite lettre, le $\ll s \gg$ au mot « pouvoirs » dans le sous-titre « culture et pouvoirs ». Ce «s $»$ ne me semble pas seulement renvoyer à la succession chronologique de ceux qui ont exercé le pouvoir politique sur un siècle en Russie et en Union soviétique (entre 1917 et 2017), mais aussi à l'aspect protéiforme de ce « pouvoir », que l'A. se refuse de naturaliser sur le mode bien connu de « l'artiste et le pouvoir ». Le pouvoir dont il est question ici, avec les contraintes qu'il peut faire peser ou le contrôle qu'il peut exercer sur les individus, est multiple : il est bien entendu politique, culturel, social, mais c'est aussi un pouvoir plus archaïque, à savoir le pouvoir clanique (comme l'indique le titre) et familial. La famille est au cœur de l'ouvrage, avec ses mensonges, ses dénis et ses non-dits, ses secrets et ses effacements, ses stratégies d'entraide, ses liens de solidarité, mais aussi ses compromissions, ses reniements, ses petits arrangements et ses grandes trahisons - rappelons que de façon on ne peut plus symbolique, la carrière littéraire de Sergueï Mikhalkov a été lancée vers le milieu des années 1930 par une chanson sur Pavlik Morozov, le petit pionnier qui a dénoncé son père, et dont le mythe a érigé la trahison familiale en vertu. L'A. clôt d'ailleurs son ouvrage sur une autre trahison paternelle : celle qui est mise en scène dans le film Citadelle de Nikita Mikhalkov, à travers le personnage de Kotov.

Le « conte russe » élaboré par la famille Mikhalkov-Kontchalovski (et l'ouvrage montre bien les diverses étapes de cette élaboration, ainsi que ses très nombreuses réécritures) ressortit en fait à plusieurs genres : il tient de la mythologie (avec un ancêtre fondateur prestigieux en la personne du peintre Vassili Sourikov, voire même Pouchkine si l'on en croit l'arbre généalogique reconstitué par Nikita Mikhalkov), de la fresque historique, du roman d'espionnage, de la chronique politique, du thriller financier qui met en scène des «papis flingueurs » et surtout de la saga familiale, une saga à multiples rebondissements qu'on lit avec autant de plaisir que son auteure a sans doute pris à l'écrire.

Aux plaisirs de lecture non négligeables que procure toute bonne saga s'ajoutent les plaisirs plus intellectuels et plus subtils offerts par l'étude universitaire. Le livre se présente comme un travail d'investigation très fouillé et très détaillé, qui 
puise à des sources diversifiées, souvent de première main. Ces sources sont sans cesse confrontées les unes aux autres dans une entreprise qui relève presque de la maïeutique ou, dans tous les cas, du dévoilement des mensonges familiaux, sociaux ou étatiques. L'A. analyse ainsi les usages du discours par les membres du clan Mikhalkov qu'elle confronte à leurs pratiques, le hiatus entre les premiers et les secondes se situant au cœur même du «contre-récit » et du processus de dévoilement.

Plonger à la suite des Mikhalkov dans «l'histoire politique et sociale de la Russie, soviétique et post-soviétique », c'est interroger les catégories de l'adaptation aux normes et aux codes culturels, les catégories du conformisme et du compromis (mais aussi de leurs antithèses incarnées dans le livre par le parcours d'un autre cinéaste: Andreï Tarkovski), c'est interroger enfin les modes de survie en régime totalitaire ou autoritaire. Autant d'éléments qui peuvent expliquer en partie l'extraordinaire longévité des relations entre le clan et le pouvoir d'État sur plus de trois générations. Car si le pouvoir est protéiforme et marqué par la duplicité, les membres du clan Mikhalkov le sont eux aussi - et ceci jusqu'au maquillage de leur nom grâce au déplacement d'un accent tonique. Leurs habitudes de travestissement, maintes fois relevées par l'A., peuvent être envisagées comme un héritage comportemental conditionné par les conditions d'existence en URSS: la question de la double, voire de la triple identité qui implique que la personne publique soit ajustée à un ensemble de règles de comportement et à des schémas de pensée qui peuvent contredire ceux de la personne privée. Mais ces habitudes peuvent aussi être considérées comme une sorte de bréviaire familial, car le dédoublement schizophrénique est finalement antérieur à la période de la Terreur chez les Mikhalkov, qui ont toujours cultivé les identités multiples. C'est là une question fascinante, qui rejoint les différentes thèses des sociologues sur le « type humain » qui aurait été généré par les pouvoirs totalitaires, mais qui renvoie aussi à des questions d'ordre psychologique tout aussi importantes. Il semble exister dans le clan une forme de psyché familiale ou d'appareil psychique familial, comme matrice des futurs individus qui la constitueront. Ici, la famille apparaît bien comme un intermédiaire, un espace tiers entre la réalité psychique individuelle et la réalité sociale. Autrement dit, chez les Mikhalkov, aussi bien les facultés d'adaptation que la propension aux personnalités multiples trouvent leur socle dans deux formes d'institution : l'institution familiale et l'institution sociopolitique, sans cesse mises en regard l'une de l'autre.

Enfin, le dernier apport important du livre réside dans l'examen minutieux de la façon dont l'histoire russe et soviétique, sa réalité matérielle et spirituelle ont pu être réfléchis par les «objets culturels » élaborés par les différents membres de la famille Mikhalkov : œuvres littéraires, scéniques ou visuelles, qui sont autant de 
SLOVO

328 Les Voyages lointains des écrivains polonais $\left(\mathrm{XX}^{\mathrm{e}}-\mathrm{XXI}^{\mathrm{e}}\right.$ siècles $)-\mathrm{n}^{\circ} 51$

formes de discours du clan sur lui-même et sur le monde. L'ouvrage de Cécile Vaissié propose une nouvelle mise en perspective et un décryptage de ces productions artistiques qui entrent en dialogue les unes avec les autres et qui sont analysées en fonction de leurs contributions à l'élaboration $\mathrm{du}$ 《 grand récit » familial et/ ou patriotique. Sergueï Mikhalkov rédige surtout le roman de la grande famille soviétique, qui s'est substituée à la famille traditionnelle à partir des années 1930, allant même jusqu'à réécrire « l'histoire intellectuelle et sociale d'un pays » dont il fut l'un des principaux édificateurs culturels. Ses deux fils, Andreï Kontchalovski et Nikita Mikhalkov, apportent quant à eux leur contribution filmique à l'écriture et la réécriture de la légende du clan : un clan pour qui l'identité familiale se confond de plus en plus avec une identité nationale vécue sur le mode slavophile de l'opposition à un Occident forcément décadent et malintentionné, dans une sorte de caricature de l'idée russe [russkaja ideja] des idéologues du XIX ${ }^{\mathrm{e}}$ siècle. Cette réécriture s'effectue selon les règles du «double code » pour l'aîné, à qui sa « plasticité » permet d'exister en tant qu'artiste à la fois dans la norme russo/soviétique et en dehors de cette norme, et selon des codes plus personnels et plus tragiques pour le cadet, dans le sens où les renvois directs ou symboliques au clan, à ses multiples ambiguïtés, ses secrets, ses dénis ou ses traumatismes sont sans doute plus nombreux. Les conflits intrapsychiques et intrafamiliaux du réalisateur suscitent in fine une réponse esthétique et visuelle qui me semble tout à fait gogolienne : l'écroulement du mythe (ou du conte russe) dans le grotesque, avec le film Citadelle. Les pages que Cécile Vaissié consacre à ce dernier opus autour du motif du « reniement du père » montrent indirectement que dans toute œuvre d'art (pour autant que Citadelle en soit une), il y a quelque chose qui échappe au contrôle de son créateur et qui « parle » malgré lui.

La conclusion, qui réaffirme avec force la relation complexe, tissée au fil des 360 pages de l'ouvrage, entre les discours produits par l'individu et la famille, et ceux produits par la société, les productions artistiques et les pouvoirs, est de ce point de vue tout à fait convaincante. Comme le stipule l'A., les œuvres des Mikhalvov peuvent et doivent être lues comme l'émanation de la culture du pays qui les a vues naître (page 338, Cécile Vaissié cite Dmitri Bykov pour qui le film Confrontation de Nikita Mikhalkov, dans son absence de logique, de goût et de cohérence, est « une expression esthétique absolument adéquate de l'époque de Poutine »). En effet, les films de l'un et l'autre des deux frères reflètent (ou plutôt diffractent) en profondeur, et dans leurs codes esthétiques mêmes, la réalité historique de l'URSS et de la Russie, avec ses peurs, ses crimes, ses séductions, ses retournements et ses manipulations.

$\mathrm{Au}$ nombre des quelques regrets suscités par l'ouvrage, je citerai l'ordre chronologique adopté par l'A. qui provoque un assez grand nombre de répétitions, 
et le fait que ne soit pas mis plus en avant un élément qui ne manque pas de frapper le lecteur de l'époque post-\#Me-too, à savoir la place plus que subalterne occupée par les femmes dans ce conte russe, qui est un conte masculin et patriarcal au sens fort du terme (c'est Nikita Mikhalkov affirmant en 2011 qu'il manque à la société russe « un principe masculin »). Quand les femmes ne sont pas effacées, elles sont instrumentalisées et parfois même réifiées, à l'instar des épouses ou des maîtresses d'Andreï Kontchalovski, par exemple, ou de la figure de la «mère ». La structure familiale ou clanique est marquée par des réflexes patriarcaux caractéristiques de la survivance actuelle en Russie d'un certain type de relations familiales établies par le Domostroï. D'un point de vue sociologique, ce que montre en creux l'histoire des Mikhalkov, leur « conte russe », c'est que malgrél'égalité affichée, les femmes n'ont que très peu accès en URSS comme en Russie contemporaine au pouvoir politique, culturel et économique. Enfin, les entretiens que l'A. a utilisés pour rédiger son livre ont presque exclusivement eu lieu avec des membres féminins de la famille, ce qui me semble également très significatif: Cécile Vaissié n'a pas eu accès aux membres masculins, c'est-à-dire à ceux qui contrôlent le discours de la story... et dont le refus témoigne d'un comportement paranoïaque face à ce qui peut-être vécu comme une « infiltration » dans l'histoire et les secrets du clan. 